\title{
Charge Density Waves in Small Metallic and Superconducting Rings
}

\author{
M.M. Maśka, K. Czajka, M. Mierzejewski and Ż. Śledé \\ Institute of Physics, University of Silesia \\ Uniwersytecka 4, 40-007 Katowice, Poland
}

\begin{abstract}
Experimental results concerning persistent currents in small rings threaded by a magnetic flux do not agree with theoretical predictions, especially for experiments performed in diffusive regime. This suggests important role of disorder in these experiments. In this paper we demonstrate how impurities present in ring modify the persistent current by generating or enhancing charge density waves. The electronic correlations are taken into account for both repulsive as well as attractive electron-electron interaction. The calculations are carried out for one-dimensional rings consisting of up to 12 lattice sites using Lanczös exact diagonalization approach, and for finite-width much larger rings using the Bogolyubov-de Gennes equations.
\end{abstract}

PACS numbers: 73.23.Ra, 74.78.--w

\section{Introduction}

Unusual properties of small (super)conducting systems have been predicted many years ago. Most of them manifest itself at low temperatures and they originate from two aspects of the new quantum regime. First, the phase coherence length of the electron is comparable to the system size, and therefore, the interference effects are important. Second, the spacing of energy levels can be larger than $k_{\mathrm{B}} T$. One of the most surprising results is the presence of a nonzero equilibrium current that circulates when a normal-metal ring is threaded by a magnetic flux $\Phi$. At zero temperature, this current is carried by the ground-state itself, therefore, it cannot decay. This effect was pointed out by Büttiker et al. [1] in 1983, and was extended by Büttiker [2]. It originates from the new boundary conditions imposed by the magnetic flux [3]. As a result, the persistent current, as well as all other thermodynamic properties of the system, are periodic with the flux, with periodicity $\Phi_{0}=h c / e$. In 1990 Lévy et al. have found experimental evidence for this phenomenon [4]. The authors have investigated the magnetic response of an assembly of $10^{7}$ copper rings using standard SQUID magnetometry 
and found that only the event harmonics of the mean current do not average to zero. The current that they measured is higher than the predicted one by more than an order of magnitude. Similar results were recently obtained for an assembly of $10^{5}$ silver rings [5]. The first experiment on a single gold loop was done by Chandrasekhar et al. in 1991 [6]. The measured current oscillates with the period $\Phi_{0}$ and its amplitude is higher than the theoretical value by more than 2 orders of magnitude. Experiment was also carried out on a system consisting of 30 gold rings [7], indicating higher than theoretically predicted current with both $\Phi_{0}$ and $\Phi_{0} / 2$ components. All the above mentioned experiments were performed on rings in diffusive regime. On the contrary, the amplitude of the persistent current measured on nearly ballistic GaAs ring remains in agreement with the theoretical prediction [8]. It suggests that disorder plays an important role in these results. From theoretical point of view disorder should reduce the amplitude of the current $I=I_{0} l / L$, where $I_{0}$ is the current in a perfect $1 \mathrm{D}$ ring, $l$ is the elastic mean free path and $L$ is the perimeter of the loop. On the other hand, Ambegaokar and Eckern showed that the electron-electron interactions may enhance the current [9]. There exist many other theoretical attempts to explain the role of both disorder and Coulomb correlations in the phenomenon of persistent current in small metallic rings. Unfortunately, the results are still ambiguous.

In this article we study how disorder affects the properties of a small ring with correlated electrons. However, in contradiction to many other approaches, we do not average over realizations of the disorder. Instead, we separately analyze different impurity configurations. The analyses are performed for repulsive, as well as for attractive interacting electrons on $1 \mathrm{D}$ and finite-width rings.

\section{One-dimensional ring}

The starting point is a 1D ring with diagonal disorder described by the Hubbard Hamiltonian

$$
H=-t \sum_{\langle i, j\rangle, \sigma} \mathrm{e}^{\mathrm{i} \theta_{i j}} a_{i \sigma}^{\dagger} a_{j \sigma}+\sum_{i} w_{i}\left(n_{i \uparrow}+n_{i \downarrow}\right)+U \sum_{i} n_{i \uparrow} n_{i \downarrow} .
$$

Here, $a_{i \sigma}^{\dagger}\left(a_{i \sigma}\right)$ creates (annihilates) an electron on site $i$ with spin $\sigma, U$ is the on-site electron-electron interaction, $n_{i \sigma}=a_{i \sigma}^{\dagger} a_{i \sigma}, t$ is the nearest-neighbor hopping integral in the absence of magnetic field $(t>0)$, and $\mathrm{e}^{\mathrm{i} \theta_{i j}}$ is the Peierls phase factor that describes the orbital response of the system to an external magnetic field

$$
\theta_{i j}=\frac{2 \pi}{\Phi_{0}} \int_{\boldsymbol{R}_{j}}^{\boldsymbol{R}_{i}} \boldsymbol{A} \cdot \mathrm{d} \boldsymbol{l} .
$$

The second term on the right-hand side (RHS) of Eq. (1) describes the diagonal disorder present in the system, with $w_{i}$ being the potential of an impurity at site $i$. The Hamiltonian (1) has exactly been diagonalized with the help of the Lanczös algorithm. At zero temperature the flux-induced current $I$ is calculated 
as $I=-\mathrm{d} E_{0} / \mathrm{d} \Phi$, where $E_{0}$ is the ground state energy and $\Phi$ is the magnetic flux piercing the ring. At finite temperature one should use the free energy $\mathcal{F}$ instead of the ground state energy $E_{0}$. Unfortunately, the Lanczös method gives only a few lowest eigenenergies and, therefore, the calculations are restricted to relatively low temperatures. In the absence of impurity both increasing temperature and increasing amplitude of interactions reduce the current. This result holds independently of the sign of the on-site interaction $U$. The situation changes when impurities are introduced into the system. The influence of a single impurity is different for attractive and repulsive interactions. Namely, for $U<0$ the presence of the impurity reduces the current, whereas for $U>0$ the maximum current occurs for finite value of the impurity potential. The overall dependence of the maximum value of the current on both the interaction and impurity potential is presented in Fig. 1. Such a behavior can be understood taking into account that the impurity plays the role of a pinning center for charge density waves (CDW). In the case of attractive electron-electron interaction even the pure system is unstable towards formation of the CDW. An impurity enhances this tendency further, reducing the current. On the other hand, in the absence of the electronic correlations an impurity itself produces Friedel's oscillations, i.e., the CDW in the vicinity of the impurity. These oscillations lead to a reduction of the current, which can be seen in Fig. 1: for $U=0$ the maximum current corresponds to $W=0$. For finite $W$, independently of its sign, the current is reduced. In this regime, repulsive electron-electron interaction suppresses Friedel's oscillations, which results in a partial restoration of the current, that achieves its maximum amplitude for $U \approx|W|$.

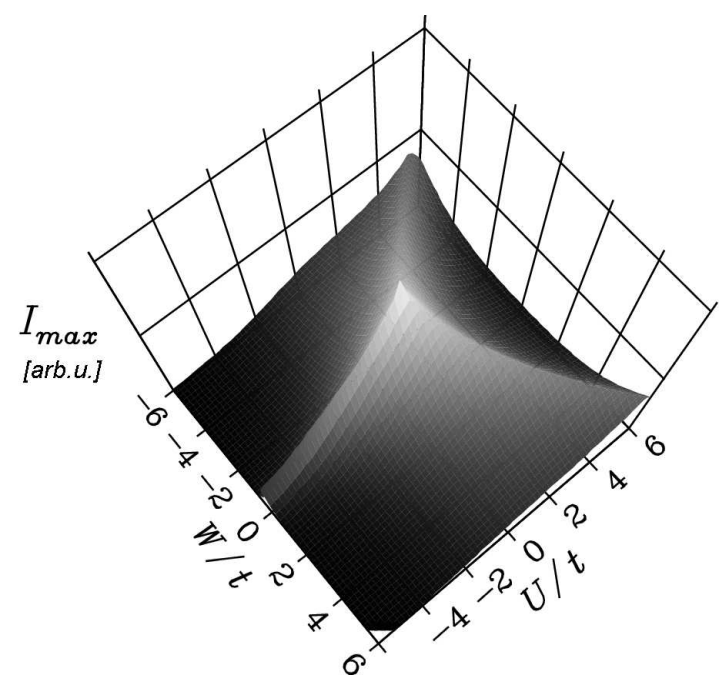

Fig. 1. Dependence of the maximum current on the electron-electron interaction $(U)$ and the impurity potential $(W)$ for a 6 -sites ring with a single impurity. 
The role of impurities in formation/enhancement of the CDW is more pronounced in the presence of two impurities, when the density waves pinned by impurities interfere. Figure 2 demonstrates how the maximum current depends on the distance $d$ between the impurities in 12-site ring. Results for both attractive and repulsive interaction are presented. The oscillatory nature of this dependence results from the interference of the CDW enhanced $(U<0)$ or formed $(U>0)$ in the vicinity of these two impurities. When the number of lattice constants between the impurities is even (odd) the overall CDW is enhanced (reduced), which leads to the reduction (enhancement) of the current. Figure 3 shows the flux dependence of the current for various impurity configurations. Let us note that the strongest
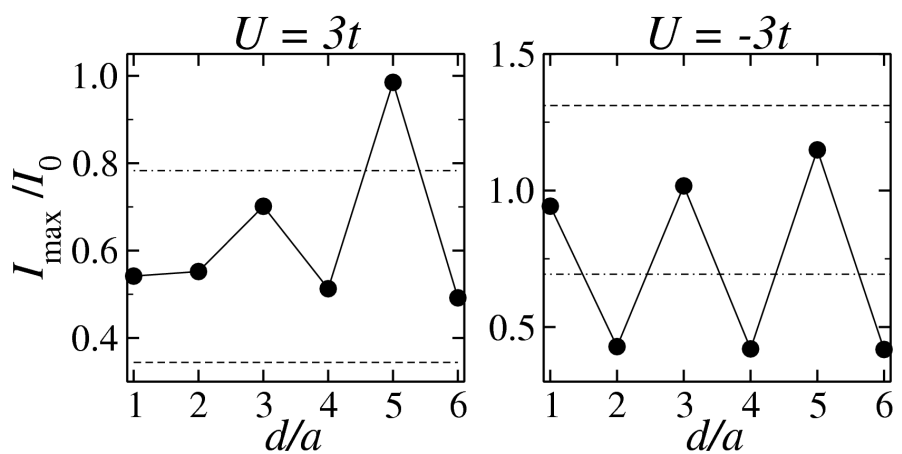

Fig. 2. Dependence of the maximum current $I_{\max }$ on the distance $d$ between impurities in 12-site ring for repulsive (left panel) and attractive (right panel) interactions. The horizontal lines indicate $I_{\max }$ in the absence of impurity (dashed line) and in the presence of a single impurity (dotted-dashed line). $I_{0}=t / \Phi_{0}$ and $a$ is the lattice constant.

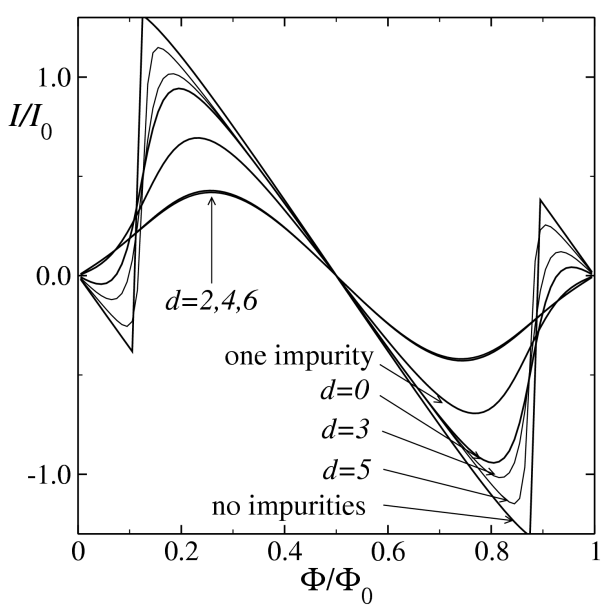

Fig. 3. Persistent current in 12-site ring as a function of the applied magnetic flux for various disorder realizations. Notation " $d=n$ " means that there are two impurities separated by $n$ lattice constants. $U=-2 t$ and $W=0.5 t$ has been assumed. 
suppression of the current takes place for $d=2,4,6$, i.e., in the cases, when the density waves pinned by different impurities remain in phase.

For three or more impurities the resultant influence also depends on distances between the impurities. In particular, when most of these distances are even multiples of the lattice constant the CDW is enhanced and the current is strongly reduced.

\section{Finite-width ring}

In this section we analyze how the impurity-generated or impurity-enhanced CDW affects properties of rings of finite width. Since the Lanczös method is applicable only to relatively small systems, consisting of up to several lattice sites, here we have to abandon this exact method. Instead, we solve the Bogolyubov-de Gennes equations (BdG) [10]. In order to do that, we have to decouple the interaction term in the Hamiltonian (1):

$$
U \sum_{i} n_{i \uparrow} n_{i \downarrow} \simeq U \sum_{i}\left(\left\langle n_{i \uparrow}\right\rangle n_{i \downarrow}+n_{i \uparrow}\left\langle n_{i \downarrow}\right\rangle\right)+U \sum_{i} \Delta_{i}^{\mathrm{SC}} a_{i \uparrow}^{\dagger} a_{i \downarrow}^{\dagger}+\text { h.c. }
$$

The first term on the RHS of Eq. (3) is responsible for the formation of CDW, $\Delta_{i}^{\mathrm{SC}}=\left\langle a_{i \downarrow} a_{i \uparrow}\right\rangle$ denotes local superconducting order parameter and is different from zero only for $U<0$. After the decoupling the Hamiltonian can be diagonalized by means of the BdG equations. See, e.g., [11, 12] for the details. The solution gives us full description (at the mean-field level) of the system under investigation as a function of the applied flux. In particular, we obtain the amplitude of the persistent current and spatial distributions of the CDW and superconducting order parameters. As an example Fig. 4 shows the current distribution in $4 \times 30$ ring with two impurities. One can see that the distribution is modified only in

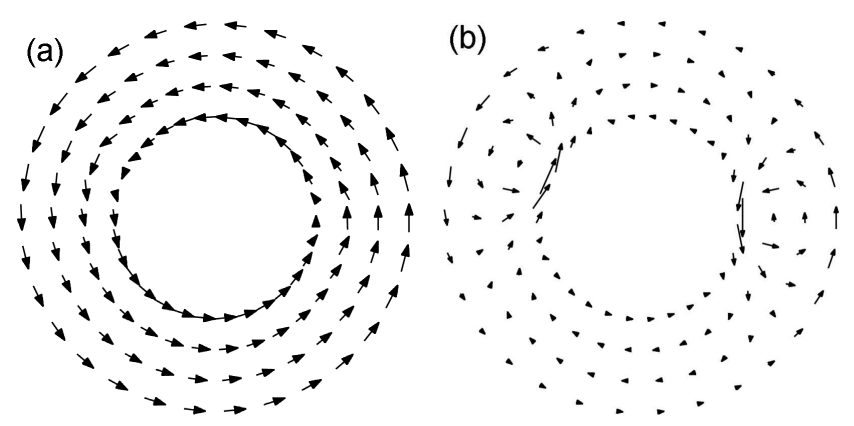

Fig. 4. (a) Current distribution in $4 \times 30$ ring with two impurities. (b) Difference between current distribution in clean ring and ring with two impurities (not in scale).

the close neighborhood of the impurities. As a result the persistent current is not as strongly modified as in the $1 \mathrm{D}$ case. On the other hand, the CDW that is enhanced around impurities, strongly modifies other properties of the ring. Figure 5 shows spatial distribution of CDW and superconducting order parameters 


\section{(a)}

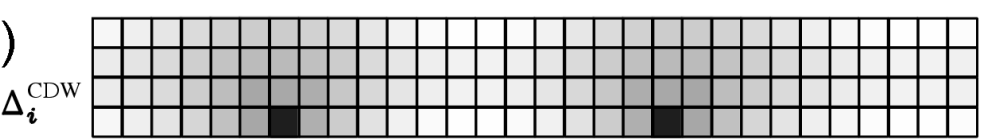

$\Delta_{i}^{\mathrm{SC}}$

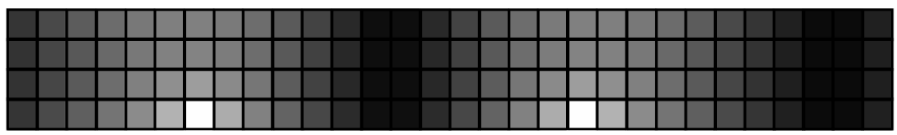

$\Delta_{i}$

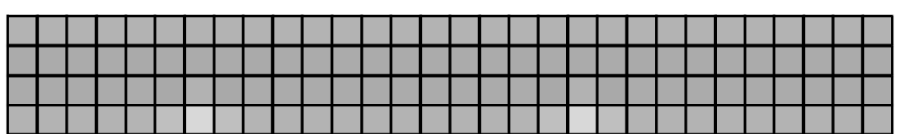

(b)

$\Delta_{i}^{\mathrm{CD}}$

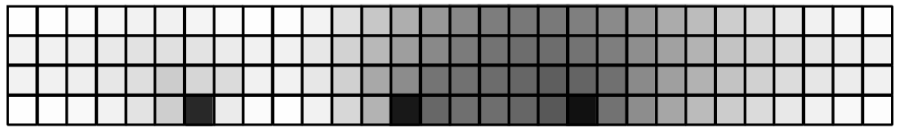

$\Delta_{i}^{\mathrm{SC}}$

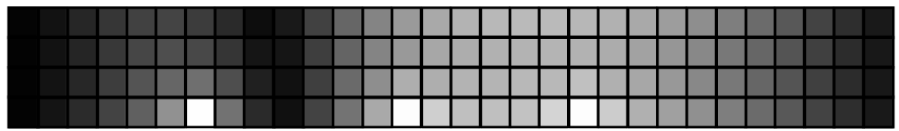

$\Delta_{i}$

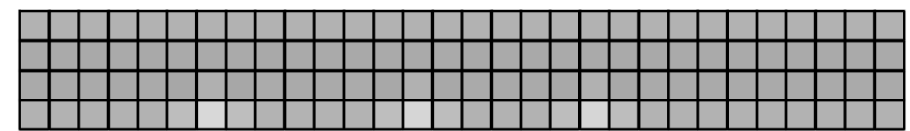

Fig. 5. Spatial distribution of the CDW order parameter $\left(\Delta_{i}^{\mathrm{CDW}}\right)$, the superconducting order parameter $\left(\Delta_{i}^{\mathrm{SC}}\right)$, and $\Delta_{i} \equiv \sqrt{\left(\Delta_{i}^{\mathrm{CDW}}\right)^{2}+\left(\Delta_{i}^{\mathrm{SC}}\right)^{2}}$. Dark squares indicate regions of large amplitude of the order parameter. See text for explanation.

calculated for $4 \times 30$ ring with pairing interactions $(U<0)$. These strips represent cut and unfolded rings. The upper three stripes (Fig. 5a) describe a ring with two impurities for which the pinned density waves are out of phase. The suppression of the superconducting order parameter occurs only in the vicinity of the impurities. The third stripe shows $\Delta_{i} \equiv \sqrt{\left(\Delta_{i}^{\mathrm{CDW}}\right)^{2}+\left(\Delta_{i}^{\mathrm{SC}}\right)^{2}}$ and this parameter is almost constant, except the sites occupied by impurities. This illustrates the competition between superconductivity and CDW phase. The next three stripes (Fig. 5b) show a ring with three impurities. In this case density waves pinned by two of the impurities are in phase, whereas the third one is out of phase. In such a configuration the region of enhanced CDW order, and consequently the region of reduced superconducting order, is much larger. $\Delta_{i}$ also in this case is almost constant over the whole ring.

\section{Summary}

We have presented numerical analysis of the nanoscopic ring pierced by external magnetic field. A large number of factors affecting the properties of the ring has been taken into account. We have demonstrated how imperfectness modifies properties of the ring in the presence of electronic correlations. In particular, 
we have shown how a specific realization of the disorder enhances or reduces the persistent current through the suppression or enhancement of the CDW. These investigations are important mainly because of the discrepancy between experimental results and theoretical predictions.

\section{References}

[1] M. Büttiker, Y. Imry, R. Landauer, Phys. Lett. A 96, 365 (1983).

[2] M. Büttiker, Phys. Rev. B 32, 1846 (1985).

[3] C. Byers, C.N. Yang, Phys. Rev. Lett. 7, 46 (1961).

[4] L.P. Lévy, G. Dolan, J. Dunsmuir, H. Bouchiat, Phys. Rev. Lett. 64, 2074 (1990).

[5] R. Deblock, R. Bel, B. Reulet, H. Bouchiat, D. Mailly, Phys. Rev. Lett. 89, 206803 (2002).

[6] V. Chandrasekhar, R.A. Webb, M.J. Brady, M.B. Ketchen, W.J. Gallagher, A. Kleinsasser, Phys. Rev. Lett. 67, 3578 (1991).

[7] E.M.Q. Jariwala, P. Mohant, M.B. Ketchen, R.A. Webb, Phys. Rev. Lett. 86, 1594 (2001); Phys. Rev. Lett. 86, 3124 (2001).

[8] D. Mailly, C. Chapetier, A. Benoît, Phys. Rev. Lett. 70, 2020 (1993).

[9] V. Ambegaokar, U. Eckern, Phys. Rev. Lett. 65, 381 (1990).

[10] P.G. de Gennes, Superconductivity of Metals and Alloys, W.A. Benjamin, New York 1966.

[11] M.M. Maśka, M. Mierzejewski, Phys. Rev. B 68, 024513 (2003).

[12] K. Czajka, M.M. Maśka, M. Mierzejewski, Ż. Śledź, Phys. Rev. B 72, 035320 (2005). 